\title{
Rake Receiver Detection of Adaptive Modulation Aided CDMA over Frequency Selective Channels
}

\author{
Byoung-Jo Choi and Lajos Hanzo ${ }^{1}$
}

\author{
Dept. of ECS, University of Southampton, SO17 1BJ, UK. \\ Tel: +44-23-8059-3125, Fax: +44-23-8059-4508 \\ Email: $1 \mathrm{~h}^{1}$ @ecs.soton.ac.uk, http://www-mobile.ecs.soton.ac.uk
}

\begin{abstract}
A closed form Bit Error Ratio (BER) formula is derived for a fixed-mode Quadrature Amplitude Modulation (QAM) scheme employing Rake receivers and receiver antenna diversity. The analysis is extended to constant power Adaptive QAM (AQAM) expressing the average BER and the average throughput as a closed form function of the modulation mode switching levels. Then, the switching levels are optimised so that the average throughput is maximised, while maintaining a given target BER. This results in a constant-BER, variable-throughput arrangement. The results show that our constant-power AQAM scheme exhibits an SNR gain of about $5 \mathrm{~dB}$ in comparison to fixed-mode QAM, when operating over a Wireless Asynchronous Transfer Mode (W-ATM) channel employing one antenna. However, the achievable throughput gain of the system over conventional fixed-mode modems is substantially reduced, as the diversity order of the receiver is increased.
\end{abstract}

\section{INTRODUCTION}

Mobile communications channels typically exhibit time variant channel quality fluctuations [1] and hence conventional fixed-mode modems suffer from bursts of transmission errors, even if the system was designed for providing a high link margin. An efficient approach to mitigating these detrimental effects is to adaptively adjust the transmission format based on the near-instantaneous channel quality perceived by the receiver, which is fed back to the transmitter with the aid of a feedback channel [2]. This scheme requires a reliable feedback link from the receiver to the transmitter and the channel quality variation should be sufficiently slow for the transmitter to be able to adapt. Hayes [2] proposed transmission power adaptation, while Cavers [3], suggested invoking a variable symbol duration scheme in response to the perceived channel quality at the expense of a variable bandwidth requirement. Since a variable-power scheme increases both the transmitted power requirements and the level of co-channel interference [4], instead variable-rate Adaptive Quadrature Amplitude Modulation (AQAM) was proposed by Steele and Webb as an alternative, employing various star-QAM constellations [4]. With the advent of Pilot Symbol Assisted Modulation (PSAM) [5] Otsuki, Sampei and Morinaga [6] employed square constellations instead of star constellations for AQAM, as a practical fading counter measure. Analysing the channel capacity of Rayleigh fading channels [7], [8], [9], Goldsmith and Varaiya showed that variable-power, variable-rate adaptive schemes are optimum,

The financial support of LGE, Korea; The CEC, Brussels and that of the EPSRC, UK is gratefully ackowledged. approaching the achievable channel capacity and they also characterised the average throughput performance of variable-power AQAM [8]. However, they also found that the additional channel capacity achieved by the variable-power assisted adaptation regime over the constant-power, variable-rate scheme is a fraction of one decibel for most types of fading channels [10], [8].

Another approach to mitigating the effects of fading is involving diversity techniques, such as space, frequency or time diversity [11, Ch 5]. Receiver antenna diversity using various combining methods [11, Ch 5] and transmitter antenna diversity employing space-time codes [12], [13] belong to the family of space-diversity schemes. By contrast, Rakereceiver [14] based Direct-Sequence (DS) Code Division Multiple Access (CDMA) [15] and Multi Carrier CDMA [16], [17], [18] can be classified as frequency-diversity assisted techniques.

Since the above two approaches are independent of each other, both Rake-receiver and antenna diversity aided schemes can be combined with AQAM. Hence, the aim of this contribution is to analyse the performance of a combined frequencyand space-diversity assisted AQAM system. Here, we employed constant-power AQAM, justified by the arguments of [4] regarding the transmit power requirements, since a variable-power scheme would result in increased co-channel interference. We note furthermore that the additional throughput gain due to applying a variable-power, rather than a constant-power scheme is small [10]. Based on the performance analysis of our combined AQAM system, we study the upper-bound performance of Rake receiver assisted AQAM employing antenna diversity.

In the next section, our system model is introduced. The Bit Error Ratio (BER) performance of Rake-receiver assisted fixedmode QAM employing antenna diversity is analysed in Section III. In Section IV, the average BER and the throughput of combined AQAM is expressed in a closed form as a function of the modulation switching thresholds. Finally, the performance of Rake receiver and receiver antenna diversity assisted AQAM employing optimum mode switching levels is presented in Section V, before concluding in Section VI.

\section{System Model}

Our Rake-receiver and $D$-antenna diversity assisted AQAM system is illustrated in Fig. 1. A band-limited equivalent low- 


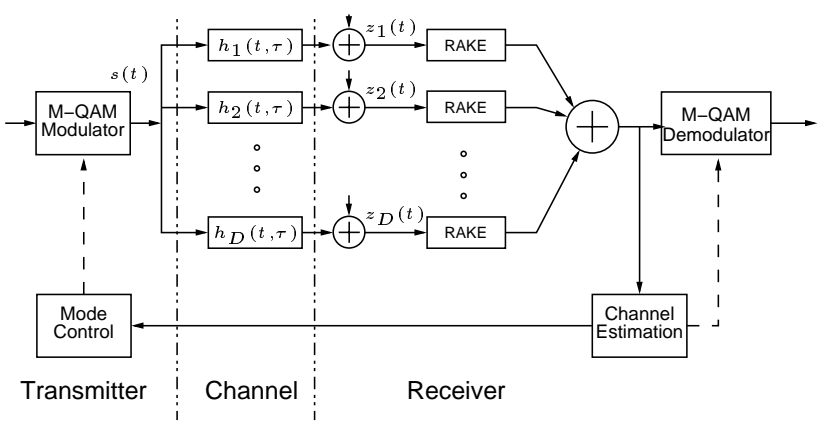

Fig. 1. Equivalent low-pass model of a $D$-th order antenna diversity based RAKE-receiver assisted AQAM system

pass $m$-ary QAM signal $s(t), S(f)=0$ for $|f|>1 / 2 W$, is transmitted over time variant frequency selective fading channels and received by a set of $D$ RAKE-receivers. Each Rakereceiver [14], [19] combines the resolvable multi-path components using Maximal Ratio Combining (MRC). The combined signals of the $D$ number of Rake-receivers seen in Fig. 1 are summed and demodulated using the estimated channel quality information. The estimated signal-to-noise ratio (SNR) $\hat{\gamma}$ is fed back to the transmitter and it is used for deciding upon the highest throughput $m$-ary square QAM modulation mode capable of maintaining the target BER. A $K$-mode adaptive modulation scheme adjust its transmit mode to mode- $k$, where $k \in\{0,1 \cdots K-1\}$, by employing $m_{k}$-ary modulation according to the estimated SNR $\hat{\gamma}$ perceived at the receiver. The mode selection rule is given by:

Choose mode $k$, when $s_{k} \leq \hat{\gamma}<s_{k+1}$,

where a switching level $s_{k}$ belongs to the set $\mathbf{s}=\left\{s_{k} \mid k=\right.$ $0,1, \cdots, K\}$. The boundary switching levels are usually given as $s_{0}=0$ and $s_{K}=\infty$. The Bit Per Symbol (BPS) throughput $b_{k}$ of a modulation mode $k$ is given as $b_{k}=\log _{2}\left(\mathrm{~m}_{\mathrm{k}}\right)$ if $m_{k} \neq 0$, otherwise $b_{k}=0$. It is convenient to define the incremental BPS $c_{k}$ as $c_{k}=b_{k}-b_{k-1}$, when $k>0$ and as $c_{0}=b_{0}$, provided that $k=0$. In an effort to derive the achievable upper bound performance we assume that the channel quality is estimated perfectly and it is available at the transmitter immediately. The effects of channel estimation error and feedback delay on the performance of AQAM were studied for example in [10]. Here a 5-mode square-constellation based AQAM scheme has been studied due to the superior BER performance of Gray-mapped square QAM constellations in comparison to other $m$-ary techniques [21]. The parameters of this 5-mode AQAM system are summarised in TABLE I.

The low-pass equivalent impulse response of the channel between the transmitter and the $d$-th antenna, $d=1,2, \cdots, D$, may be represented as [19]:

$$
h_{d}(t, \tau)=\sum_{n=1}^{N} h_{d, n}(t) \delta\left(\tau-\frac{n}{W}\right)
$$

where $\left\{h_{d, n}(t)\right\}$ is a set of independent complex valued stationary random processes. The maximum number of resolvable multi-path components $N$ is given by $\left\lfloor I^{\prime}{ }_{m} W\right\rfloor+1$, where $I^{\prime}{ }_{m}$ is the multi-path delay spread of the channel [19]. Hence, the low-pass equivalent received signal $r_{d}(t)$ at the $d$-th antenna, $d=1,2, \cdots, D$, can be represented as:

$$
r_{d}(t)=\sum_{n=1}^{N} h_{d, n}(t) s\left(t-\frac{n}{W}\right)+z_{d}(t),
$$

where $z_{d}(t)$ is a zero mean Gaussian random process having a two-sided power spectral density of $N_{o} / 2$. Let us assume that

TABLE I

THE PARAMETERS OF 5-MODE AQAM SYSTEM

\begin{tabular}{c||c|c|c|c|c}
\hline \hline$k$ & 0 & 1 & 2 & 3 & 4 \\
\hline$m_{k}$ & 0 & 2 & 4 & 16 & 64 \\
\hline$b_{k}$ & 0 & 1 & 2 & 4 & 6 \\
\hline$c_{k}$ & 0 & 1 & 1 & 2 & 2 \\
\hline mode & No Tx & BPSK & QPSK & 16QAM & 64QAM \\
\hline \hline
\end{tabular}

the fading is sufficiently slow or $(\Delta t)_{c} \ll T$, where $(\Delta t)_{c}$ is the channel's coherence time [1] and $T$ is the signaling period. Then, $h_{d, n}(t)$ over a signaling period $T$ can be simplified to $h_{d, n}(t)=\alpha_{d, n} e^{j \phi_{d, n}}$, where the fading magnitude $\alpha_{d, n}$ is assumed to be Rayleigh distributed and the phase $\phi_{d, n}$ is assumed to be uniformly distributed.

\section{BER ANALYSIS OF $m$-ARY QAM}

An ideal RAKE receiver [14] combines all the signal powers scattered over $N$ paths in an optimal manner so that the instantaneous Signal-to-Noise Ratio (SNR) per symbol at the RAKE receiver's output can be maximised [19]. The noise at the RAKE receiver's output is known to be Gaussian [19]. The SNR, $\gamma_{d}$, at the $d$-th ideal RAKE receiver's output in Fig. 1 is given as [19]:

$$
\gamma_{d}=\sum_{n=1}^{N} \gamma_{d, n},
$$

where $\gamma_{d, n}=E / N_{o} \alpha_{d, n}^{2}$ and $\left\{\alpha_{d, n}\right\}$ is assumed to be normalised, such that $\sum_{n=1}^{N} \alpha_{d, n}^{2}$ becomes unity. Since we assumed that each multi-path component has an independent Rayleigh distribution, the characteristic function of $\gamma_{d}$ can be represented as [19, pp 802]:

$$
\psi_{\gamma_{d}}(j v)=\prod_{n=1}^{N} \frac{1}{1-j v \bar{\gamma}_{d, n}},
$$

where $\gamma_{d, n}=E / N_{o} \mathrm{E}\left[\alpha_{\mathrm{d}, \mathrm{n}}^{2}\right]$. Let us assume further more that each of the $D$ diversity channels of Fig. 1 has the same multipath intensity profile (MIP), although in practical systems it may have a different MIP. Under this assumption, $\bar{\gamma}_{d, n}$ in (5) can be written as $\bar{\gamma}_{n}$. The total SNR per symbol, $\gamma$, at the output of the demodulator depicted in Fig. 1 is given as:

$$
\gamma=\sum_{d=1}^{D} \gamma_{d}
$$


while the characteristic function of $\gamma$, under the assumption of independent identical diversity channels, can be formulated as:

$$
\psi_{\gamma}(j v)=\prod_{n=1}^{N} \frac{1}{\left(1-j v \bar{\gamma}_{n}\right)^{D}}
$$

Applying the well-known technique of Partial Fraction Expansion (PFE) [20], $\psi_{\gamma}(j v)$ can be expressed as:

$$
\psi_{\gamma}(j v)=\sum_{d=1}^{D} \sum_{n=1}^{N} \Lambda_{d, n} \frac{1}{\left(1-j v \bar{\gamma}_{n}\right)^{d}}
$$

where the constant $\Lambda_{d, n}$ can be found by equating (7) and (8).

The PDF of $\gamma, f_{\bar{\gamma}}(\gamma)$, can be found by applying the inverse Fourier transform to $\psi_{\gamma}(j v)$ in (8), which is given, with the aid of [19, pp 781 (14-4-13)], by:

$$
f(\gamma)=\sum_{d=1}^{D} \sum_{n=1}^{N} \Lambda_{d, n} \frac{1}{(d-1) ! \bar{\gamma}_{n}^{d}} \gamma^{d-1} e^{-\gamma / \bar{\gamma}_{n}}
$$

Since we now have the PDF $f_{\bar{\gamma}}(\gamma)$ of the channel SNR, let us calculate the average BER of $m$-ary square QAM employing Gray mapping. The average BER $P_{e}$ can be expressed as [21], [19]:

$$
P_{e, k}=\int_{0}^{\infty} p_{m_{k}}(\gamma) f(\gamma) d \gamma
$$

where $p_{m_{k}}(\gamma)$ is the BER of $m$-ary square QAM employing Gray mapping over Gaussian channels [21]:

$$
p_{m_{k}}(\gamma)=\sum_{i} A_{i} Q\left(\sqrt{a_{i} \gamma}\right)
$$

where $Q(x)$ is the Gaussian $Q$-function defined as $Q(x) \triangleq$ $\frac{1}{\sqrt{2 \pi}} \int_{x}^{\infty} e^{-t^{2} / 2} d t$ and $\left\{A_{i}, a_{i}\right\}$ is a set of modulation mode dependent constants, which are given, for example, in [21], [22]. The average BER of $m$-ary QAM in our scenario can be calculated by substituting $p_{m_{k}}(\gamma)$ of (11) and $f_{\bar{\gamma}}(\gamma)$ of (9) into (10):

$$
\begin{aligned}
P_{e, k}(\bar{\gamma}) & =\int_{0}^{\infty} \sum_{i} A_{i} Q\left(\sqrt{a_{i} \gamma}\right) f(\gamma) d \gamma \\
& =\sum_{i} A_{i} P_{e}\left(\bar{\gamma} ; a_{i}\right)
\end{aligned}
$$

where each constituent BER $P_{e}\left(\bar{\gamma} ; a_{i}\right)$ is defined as:

$$
P_{e}\left(\bar{\gamma} ; a_{i}\right)=\int_{0}^{\infty} Q\left(\sqrt{a_{i} \gamma}\right) f(\gamma) d \gamma
$$

Using the similarity of $f_{\bar{\gamma}}(\gamma)$ in (9) and the PDF of the SNR of a $D$ antenna-diversity assisted Maximal Ratio Combining (MRC) system transmitting over flat Rayleigh channels [19, pp 781], the closed form solution for the component BER $P_{e}\left(\bar{\gamma} ; a_{i}\right)$ can be expressed as:

$$
\begin{aligned}
& P_{e}\left(\bar{\gamma} ; a_{i}\right) \\
& =\sum_{d=1}^{D} \sum_{n=1}^{N} \frac{1}{\sqrt{2 \pi}} \int_{0}^{\infty} \int_{\sqrt{2 \gamma}}^{\infty} e^{-x^{2} / 2} \Lambda_{d, n} \frac{\gamma^{d-1} e^{-\gamma / \bar{\gamma}_{n}}}{(d-1) ! \bar{\gamma}_{n}^{d}} d x d \gamma \\
& =\sum_{d=1}^{D} \sum_{n=1}^{N} \Lambda_{d, n}\left\{\frac{1-\mu_{n}}{2}\right\}^{d} \sum_{i=0}^{d-1}\left(\begin{array}{c}
d-1+i \\
i
\end{array}\right)\left\{\frac{1}{2}\left(1+\mu_{n}\right)\right\}^{i},
\end{aligned}
$$

where $\mu_{n} \triangleq \sqrt{\frac{a_{i} \bar{\gamma}_{n}}{2+a_{i} \bar{\gamma}_{n}}}$ and the average SNR per symbol is $\bar{\gamma}=D \sum_{n=1}^{N} \bar{\gamma}_{n}$. Substituting $P_{e}\left(\bar{\gamma} ; a_{i}\right)$ of (15) into (13), the average BER of an $m$-ary QAM Rake receiver using antenna diversity can be expressed in a closed form.

\section{PERFormance of AQAM}

In this section, we consider an ideal five-mode AQAM scheme characterised by the parameters given in TABLE I.

\section{A. Average Throughput}

The average throughput $B(\bar{\gamma}, \mathbf{s})$ expressed in terms of Bits Per Symbol (BPS) is given by [23]:

$$
B(\bar{\gamma}, \mathbf{s})=\sum_{k=0}^{K-1} b_{k} \int_{s_{k}}^{s_{k+1}} f(\gamma) d \gamma=\sum_{k=0}^{K-1} c_{k} F_{c}(\gamma),
$$

where $F_{c}(\gamma)$ is the complementary Cumulative Distribution Function $(\mathrm{CDF})$ of the instantaneous $\operatorname{SNR} \gamma$, given as:

$$
F_{c}(\gamma)=\sum_{d=1}^{D} \sum_{n=1}^{N} \Lambda_{d, n} e^{-\gamma / \bar{\gamma}_{n}} \sum_{k=0}^{d-1} \frac{\left(\gamma / \bar{\gamma}_{n}\right)^{k}}{\Gamma(k+1)},
$$

where $\Gamma(x)$ is the Gamma function [20].

\section{B. Average Bit Error Ratio}

Let us define the mode-specific average BER $P_{k}$ as:

$$
P_{k} \triangleq \int_{s_{k}}^{s_{k+1}} p_{m_{k}}(\gamma) f(\gamma) d \gamma
$$

Upon substituting $p_{m_{k}}(\gamma)$ of (11) and $f(\gamma)$ of (9) into (18), we have:

$$
\begin{aligned}
P_{k} & =\sum_{i} A_{i} \sum_{d=1}^{D} \sum_{n=1}^{N} \Lambda_{d, n} \int_{s_{k}}^{s_{k+1}} Q\left(\sqrt{a_{i} \gamma}\right) \frac{\gamma^{d-1} e^{-\gamma / \bar{\gamma}_{n}}}{(d-1) ! \bar{\gamma}_{n}^{d}} d \gamma \\
& =\sum_{i} A_{i} \sum_{d=1}^{D} \sum_{n=1}^{N} \Lambda_{d, n} P_{R}\left(s_{k}, s_{k+1}, \bar{\gamma}_{n}, d, a_{i}\right) .
\end{aligned}
$$

A closed form expression for $P_{R}\left(s_{k}, s_{k+1}, \bar{\gamma}_{n}, d, a_{i}\right)$ can be found by applying change-of-variables repeatedly, which can be expressed as:

$$
\begin{aligned}
P_{R}= & {\left[-e^{-\gamma / \bar{\gamma}_{n}} Q\left(\sqrt{a_{i} \gamma}\right) \sum_{j=0}^{d-1} \frac{\left(\gamma / \bar{\gamma}_{n}\right)^{j}}{\Gamma(j+1)}\right]_{s_{k}}^{s_{k+1}} } \\
& +\left[\sum_{j=0}^{d-1} X_{j}\left(\gamma ; \bar{\gamma}_{n}, a_{i}\right)\right]_{s_{k}}^{s_{k+1}}
\end{aligned}
$$


where $[g(\gamma)\rfloor_{s_{k}}^{u_{k+1}} \equiv g\left(s_{k+1}\right)-g\left(s_{k}\right)$ and $X_{j}$ is given by:

$$
\begin{aligned}
& X_{j}\left(\gamma ; \bar{\gamma}_{n}, a_{i}\right)= \\
& \frac{\mu_{n}^{2}}{\sqrt{2 a_{i} \pi}} \frac{\Gamma\left(j+\frac{1}{2}\right)}{\bar{\gamma}_{n}^{j} \Gamma(j+1)} \sum_{k=1}^{j}\left(\frac{2 \mu_{n}^{2}}{a_{i}}\right)^{j-k} \frac{\gamma^{k-\frac{1}{2}}}{\Gamma\left(k+\frac{1}{2}\right)} e^{-a_{i} \gamma /\left(2 \mu_{n}^{2}\right)} \\
& +\left(\frac{2 \mu_{n}^{2}}{a_{i} \bar{\gamma}_{n}}\right)^{j} \frac{1}{\sqrt{\pi}} \frac{\Gamma\left(j+\frac{1}{2}\right)}{\Gamma(j+1)} \mu_{n} Q\left(\sqrt{a_{i} \gamma} / \mu_{n}\right)
\end{aligned}
$$

where, again, $\mu_{n} \triangleq \sqrt{\frac{a_{i} \bar{\gamma}_{n}}{2+a_{i} \bar{\gamma}_{n}}}$ and $\Gamma(x)$ is the Gamma function. Then, the average BER $P_{\text {avg }}(\bar{\gamma}, \mathbf{s})$ of our adaptive modulation scheme can be represented as [23] :

$$
P_{a v g}(\bar{\gamma}, \mathbf{s})=\frac{1}{B(\bar{\gamma}, \mathbf{s})} \sum_{k=0}^{K-1} b_{k} P_{k},
$$

where the average BPS throughput $B(\bar{\gamma})$ and the mode-specific average BER $P_{k}$ are given by (16) and (19), respectively, and $b_{k}$ is the BPS for $m_{k}$-ary fixed-mode modulation scheme.

\section{Optimum switching levels}

Torrance and Hanzo [24] proposed a set of mode switching levels $\mathbf{s}$ optimised for achieving the highest average BPS throughput while maintaining the target average BER. The authors of this contribution recently proposed a set of SNRdependent mode switching levels [25], which keeps the average BER of AQAM constant, while maximising the achievable throughput. The sets of switching levels derived in [24], [25] are based on Powell's multidimensional optimisation technique [26] and hence the optimisation process may become trapped in a local minimum. In order to overcome this problem, we derived an optimum set of switching levels [27] employing the Lagrangian multiplier technique and showed that this set of switching levels results in the global optimum in a sense that the corresponding AQAM scheme obtains the maximum possible average BPS throughput, while maintaining the target average BER. In this paper we employ the globally optimised switching levels of [27] for studying the performance of the proposed Rake receiver aided and $D$-antenna diversity assisted AQAM scheme.

\section{RESUlts AND Discussions}

The associated performance results of the AQAM system are shown in Fig. 2 for transmission over the 3-path indoor W-ATM channel model of [21, pp. 476] employing a single antenna . Specifically, the W-ATM channel is a 3-path indoor channel, where the average SNR for each path is given as $\bar{\gamma}_{1}=0.79192 \bar{\gamma}$, $\bar{\gamma}_{2}=0.12424 \bar{\gamma}$ and $\bar{\gamma}_{3}=0.08384 \bar{\gamma}$. The performance depicted in Fig. 2 corresponds to the single user performance of a Rake-receiver assisted DS-CDMA system employing AQAM, i.e. without inflicting multi-user interference. Near single user performance is achievable for multiple users, when we employ an optimal multi-user detector [28]. The BER of fixed mode modems communicating over this channel were comparable to that recorded for transmission over flat Rayleigh channels using second-order antenna diversity. Fig. 2(a) and 2(b) show that

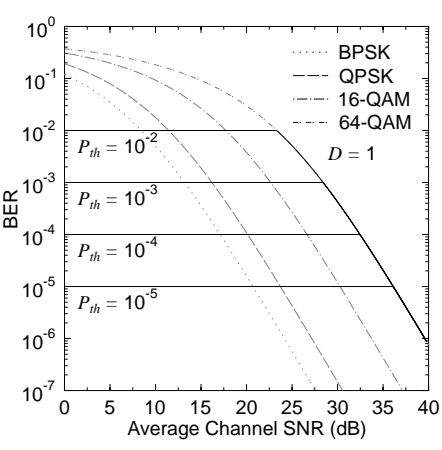

(a) BER

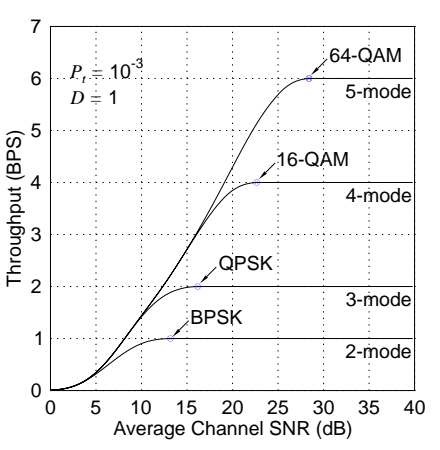

(b) Throughput: $P_{t}=10^{-3}$
Fig. 2. BER and throughput performance for transmission over the Wireless ATM channel [21] $(N=3)$ using a single antenna, i.e. $D=1$.

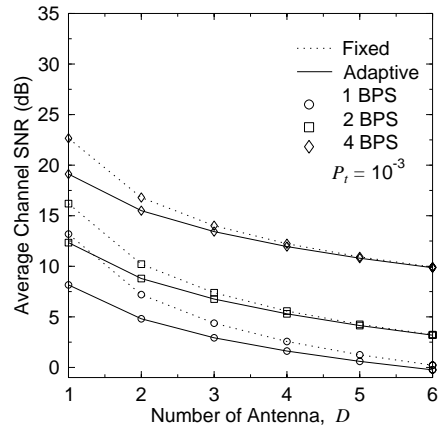

(a) SNR versus diversity order

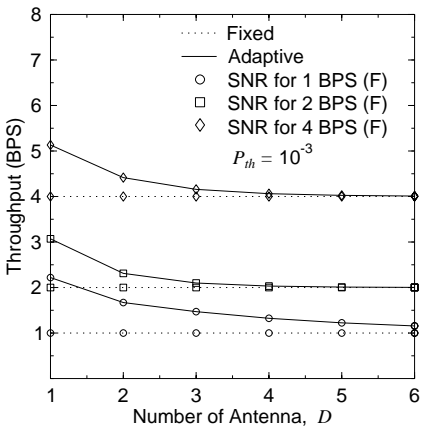

(b) Throughput versus diversity order
Fig. 3. The required SNR and the achievable BPS throughput of AQAM using different orders of antenna diversity for transmission over the Wireless ATM channel [21] $(N=3)$ at a target BER of $P_{t h}=10^{-3}$

the system maintained the required constant target BER, while increasing the average throughput, as the SNR increased.

The performance of the Rake receiver assisted AQAM scheme is shown in Fig. 3 for various antenna diversity orders together with the performance of the constituent fixed mode modems employing Rake-receivers. The SNR gain of the AQAM scheme over the fixed modems was in the range of $4 \mathrm{~dB}$ to $5 \mathrm{~dB}$, when a single antenna was used. When the number of antennas employed was higher than two, this gain was reduced below $1 \mathrm{~dB}$ compared to the fixed mode QPSK and 16QAM modems employing Rake-receivers. Fig. 3(b) shows the throughput of the Rake-receiver assisted AQAM system at the specific SNRs, where the fixed mode modems employing Rakereceivers achieved the target BER of $10^{-3}$. As seen by comparing Fig. 2(b) and Fig. 3(b), the AQAM system achieved a throughput of $2.2 \mathrm{BPS}$ at the SNR of $13.2 \mathrm{~dB}$, where a BPSK modem had a throughput of 1 BPS, provided that a single antenna was employed. As seen in Fig. 3(b) the throughput gains of the AQAM system eroded, as the diversity order was increased.

The effect of antenna diversity on the throughput of AQAM 


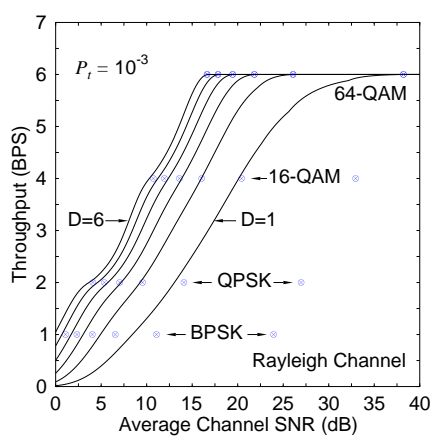

(a) Flat Rayleigh channel

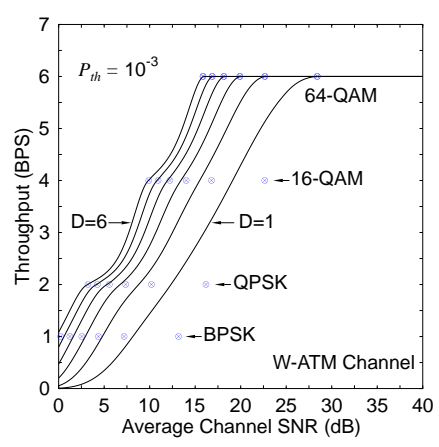

(b) W-ATM channel
Fig. 4. BPS throughput of 5-mode AQAM $\left(P_{t h}=10^{-3}\right)$. Markers represent the throughput of fixed modems. As expected, the right most markers and lines represent the single antenna $(D=1)$ scenario.

modems can be observed also in Fig. 4. The markers in the figure represent the throughput of fixed mode modems. We can observe that the markers approach the corresponding AQAMrelated lines in both graphs, as the order of antenna diversity $(D)$ is increased. The distance between the markers and lines was already shown in in Fig. 3(a). The effects of discrete-rate modulation can also be observed in Fig. 4. The second derivatives of the graphs are not monotonic. This became pronounced for the larger values of $D$, where the switching between constituent modulation modes results in the undulation observed in the BPS curve. This suggests that despite the optimised modulation switching levels, the sub-optimal effects of using only five different discrete transmission rates could not be completely eliminated.

\section{CONCLUSIONS}

The BER of AQAM systems employing Rake-receivers and antenna diversity, was analysed and a closed form BER expression was derived. The average BER and the throughput of a constant-power AQAM system employing Rake-receivers and antenna diversity were expressed in closed forms. This facilitated the optimisation of modulation switching levels. The optimised AQAM system exhibited a constant BER and an increased throughput comparable to variable-rate, variable-power MQAM systems [10]. We found that the SNR gain of AQAM modems erodes, as the order of diversity increases.

\section{REFERENCES}

[1] R. Steele and L. Hanzo, Eds., Mobile Radio Communications, IEEE Press - John Wiley \& Sons, New York, USA, 2nd edition, 1999.

[2] J. F. Hayes, "Adaptive feedback communications," IEEE Transactions on Communications, vol. 16, no. 1, pp. 29-34, 1968.

[3] J. K. Cavers, "Variable rate transmission for rayleigh fading channels," IEEE Transactions on Communications, vol. 20, pp. 15-22, February 1972.

[4] W. T. Webb and R. Steele, "Variable rate QAM for mobile radio," IEEE Transactions on Communications, vol. 43, no. 7, pp. 2223-2230, 1995.

[5] M. L. Moher and J. H. Lodge, "TCMP - a modulation and coding strategy for rician fading channels," IEEE Journal on Selected Areas in Communications, vol. 7, no. 9, pp. 1347-1355, December 1989.

[6] S. Otsuki, S. Sampei, and N. Morinaga, "Square QAM adaptive modula-
tion/TDMA/TDD systems using modulation level estimation with Walsh function," Electronics Letters, vol. 31, no. 3, pp. 169-171, February 1995.

[7] W. C. Y. Lee, "Estimate of channel capacity in Rayleigh fading environment," IEEE Transactions on Vehicular Technology, vol. 39, no. 3, pp. 187-189, August 1990.

[8] A. J. Goldsmith and P. P. Varaiya, "Capacity of fading channels with channel side information," IEEE Transactions on Information Theory, vol. 43, no. 6, pp. 1986-1992, November 1997.

[9] M. S. Alouini and A. J. Goldsmith, "Capacity of Rayleigh fading channels under different adaptive transmission and diversity-combining technique," IEEE Transactions on Vehicular Technology, vol. 48, no. 4, pp. 11651181, July 1999.

[10] A. Goldsmith and S. Chua, "Variable rate variable power MQAM for fading channels," IEEE Transactions on Communications, vol. 45, pp. 1218-1230, October 1997.

[11] W. C. Jakes Jr., Ed., Microwave Mobile Communications, John Wiley \& Sons, New York, USA, 1974.

[12] V. Tarokh, N. Seshadri, and A. Calderbank, "Space-time codes for high data rate wireless communication: Performance criterion and code construction," IEEE Transactions on Information Theory, vol. 44, no. 2, pp. 744-765, March 1998.

[13] S. M. Alamouti, "A simple transmit diversity technique for wireless communications," IEEE Journal on Selected Areas in Communications, vol. 16, no. 8, pp. 1451-1458, October 1998.

[14] R. Price and E. P. Green Jr., "A communication technique for multipath channels," Proceedings of the IRE, vol. 46, pp. 555-570, March 1958.

[15] R. L. Pickholtz, L. B. Milstein, and D. L. Schilling, "Spread spectrum for mobile communications," IEEE Transactions on Vehicular Technology, vol. 40, no. 2, pp. 312-322, May 1991.

[16] N. Yee, J-P. Linnartz, and G. Fettweis, "Multicarrier CDMA in indoor wireless radio networks," in Proceedings of IEEE PIMRC 1993, 1993, pp. $109-113$.

[17] K. Fazel and L. Papke, "On the performance of convolutionally-coded CDMA/OFDM for mobile communication system," in Proceedings of IEEE PIMRC 1993, 1993, pp. 468-472.

[18] A. Chouly, A. Brajal, and S. Jourdan, "Orthogonal multicarrier techniques applied to direct sequence spread spectrum CDMA systems," in Proceedings of IEEE GLOBECOM 1993, Houston, USA, 29 November - 2 December 1993, pp. 1723-1728.

[19] J. G. Proakis, Digital Communications, Mc-Graw Hill International Editions, 3rd edition, 1995.

[20] E. Kreyszig, Advanced engineering mathematics, John Wiley \& Sons, Inc., 7th edition, 1993.

[21] L. Hanzo, W. T. Webb, and T. Keller, Single- and Multi-carrier Quadrature Amplitude Modulation, IEEE Press-John Wiley, New York, USA, April 2000.

[22] D. Yoon, K. Cho, and J. Lee, "Bit error probability of M-ary Quadrature Amplitude Modulation," in Proceedings of IEEE VTC 2000 Fall. IEEE, September 2000, vol. 5, pp. 2422-2427.

[23] J. M. Torrance and L. Hanzo, "Upper bound performance of adaptive modulation in a slow Rayleigh fading channel," Electronics Letters, vol. 32, no. 8, pp. 718-719, 11 April 1996.

[24] J. M. Torrance and L. Hanzo, "Optimisation of switching levels for adaptive modulation in a slow Rayleigh fading channel," Electronics Letters, vol. 32, no. 13, pp. 1167-1169, 20 June 1996.

[25] B. J. Choi, M. Münster, L. L. Yang, and L. Hanzo, "Performance of Rake receiver assisted adaptive-modulation based CDMA over frequency selective slow Rayleigh fading channel," Electronics Letters, vol. 37, no. 4, pp. 247-249, February 2001.

[26] W. H. Press, S. A. Teukolsky, W. T. Vetterling, and B. P. Flannery, Numerical Recipies in C, Cambridge University Press, 1992.

[27] B. J. Choi and L. Hanzo, "Optimum mode-switching levels for adaptive modulation systems," unpublished, 2001.

[28] S. Verdú, "Minimum probability of error for asynchronous Gaussian multiple-access channel," IEEE Transactions on Communications, vol. 32, no. 1, pp. 85-96, January 1986. 